\title{
Well-Differentiated Papillary Mesothelioma of the Peritoneum Is Genetically Distinct from Malignant Mesothelioma
}

\author{
Raunak Shrestha $\left.{ }^{1,2,3}{ }^{(}\right)$, Noushin Nabavi ${ }^{1,4}{ }^{\oplus}$, Stanislav Volik ${ }^{1}$, Shawn Anderson ${ }^{1}$, \\ Anne Haegert ${ }^{1}$, Brian McConeghy ${ }^{1}$, Funda Sar ${ }^{1}$, Sonal Brahmbhatt ${ }^{1}$, Robert Bell ${ }^{1}$, \\ Stephane Le Bihan ${ }^{1}$, Yuzhuo Wang 1,2,4 ${ }^{\mathbb{D}}$, Colin Collins ${ }^{1,2, *}$ and Andrew Churg ${ }^{5, *}$ \\ 1 Vancouver Prostate Centre, Vancouver, BC V6H 3ZH, Canada; raunakman.shrestha@ucsf.edu (R.S.); \\ nabavinoushin@gmail.com (N.N.); svolik@prostatecentre.com (S.V.); sanderson@prostatecentre.com (S.A.); \\ ahaegert@prostatecentre.com (A.H.); brian.mcconeghy@ubc.ca (B.M.); fsar@prostatecentre.com (F.S.); \\ sonal.brahmbhatt2001@gmail.com (S.B.); rbell@prostatecentre.com (R.B.); \\ slebihan@prostatecentre.com (S.L.B.); ywang@bccrc.ca (Y.W.) \\ 2 Department of Urologic Sciences, University of British Columbia, Vancouver, BC V5Z 1M9, Canada \\ 3 Department of Radiation Oncology, University of California San Francisco, San Francisco, CA94143, USA \\ 4 Department of Experimental Therapeutics, BC Cancer Agency, Vancouver, BC V5Z 1L3, Canada \\ 5 Department of Pathology, Vancouver General Hospital, Vancouver, BC V5Z 1M9, Canada \\ * Correspondence: ccollins@prostatecentre.com (C.C.); achurg@mail.ubc.ca (A.C.)
}

Received: 17 May 2020; Accepted: 11 June 2020; Published: 13 June 2020

\begin{abstract}
Well-differentiated papillary mesothelioma (WDPM) is an uncommon mesothelial proliferation that is most commonly encountered as an incidental finding in the peritoneal cavity. There is controversy in the literature about whether WDPM is a neoplasm or a reactive process and, if neoplastic, whether it is a variant or precursor of epithelial malignant mesothelioma or is a different entity. Using whole exome sequencing of five WDPMs of the peritoneum, we have identified distinct mutations in EHD1, ATM, FBXO10, SH2D2A, CDH5, MAGED1, and TP73 shared by WDPM cases but not reported in malignant mesotheliomas. Furthermore, we show that WDPM is strongly enriched with C > A transversion substitution mutations, a pattern that is also not found in malignant mesotheliomas. The WDPMs lacked the alterations involving BAP1, SETD2, NF2, $C D K N 2 A / B, L A S T S 1 / 2, P B R M 1$, and SMARCC1 that are frequently found in malignant mesotheliomas. We conclude that WDPMs are neoplasms that are genetically distinct from malignant mesotheliomas and, based on observed mutations, do not appear to be precursors of malignant mesotheliomas.
\end{abstract}

Keywords: well-differentiated papillary mesothelioma; WDPM; malignant mesothelioma; DNA sequencing; mutation

\section{Introduction}

Well-differentiated papillary mesothelioma (WDPM) is a morphologically distinctive papillary proliferation of mesothelial cells that is most commonly encountered as an incidental finding in the peritoneal cavity, and less often in the pleural cavity, pericardium, and tunica vaginalis. These lesions may be single or multiple but by definition do not invade the underlying stroma and usually behave in a benign or indolent fashion, sometimes persisting for many years [1]. However, the nature of WDPM is disputed, with theories ranging from a reactive non-neoplastic process to a benign tumor, to a variant and/or precursor of epithelial malignant mesotheliomas [2]. To add further confusion, unequivocal invasive malignant mesotheliomas can have areas that mimic WDPM. Since malignant mesotheliomas are aggressive tumors, the distinction from WDPM is important, but WDPMs are 
sometimes treated with debulking cytoreductive surgery followed by hyperthermic intraperitoneal chemotherapy (HIPEC) as if they were mesotheliomas [3].

Genome-wide sequencing analyses of malignant mesotheliomas have revealed frequently observed genomic aberrations such as loss of function mutation and/or copy number alterations/deletion of BAP1, SETD2, CDKN2A, and NF2 [4-6]. Studies analyzing WDPM using DNA sequencing technology are limited. Case studies have reported WDPMs with somatic mutation of E2F1 [7], heterozygous loss of NF2 [8], and germline BAP1 mutation [9], which if correct would suggest that they may be variants of malignant mesothelioma. Nevertheless, using immunohistochemistry (IHC) and fluorescence in situ hybridization (FISH), Lee et al. demonstrated that, unlike in malignant mesothelioma, both BAP1 and CDKN2A are intact and respective proteins are expressed in WDPMs [10]. More recently, Stevers et al. [11] performed genomic profiling of 10 WDPMs and found that they harbored TRAF7 or CDC42 mutually exclusive missense mutations.

To shed further light on this question we performed an extensive genomic characterization of a cohort of five WDPMs of the peritoneum.

\section{Results}

\subsection{Histopathological Features of WDPM}

We assembled a cohort of five incidentally identified WDPM cases in the peritoneum detected during surgery for another process and all were solitary lesions. All of these five cases had the typical features described for WDPM [12], i.e., a papillary architecture with a single layer of covering bland mesothelial cells and myxoid cores in the papillae (Figure 1).
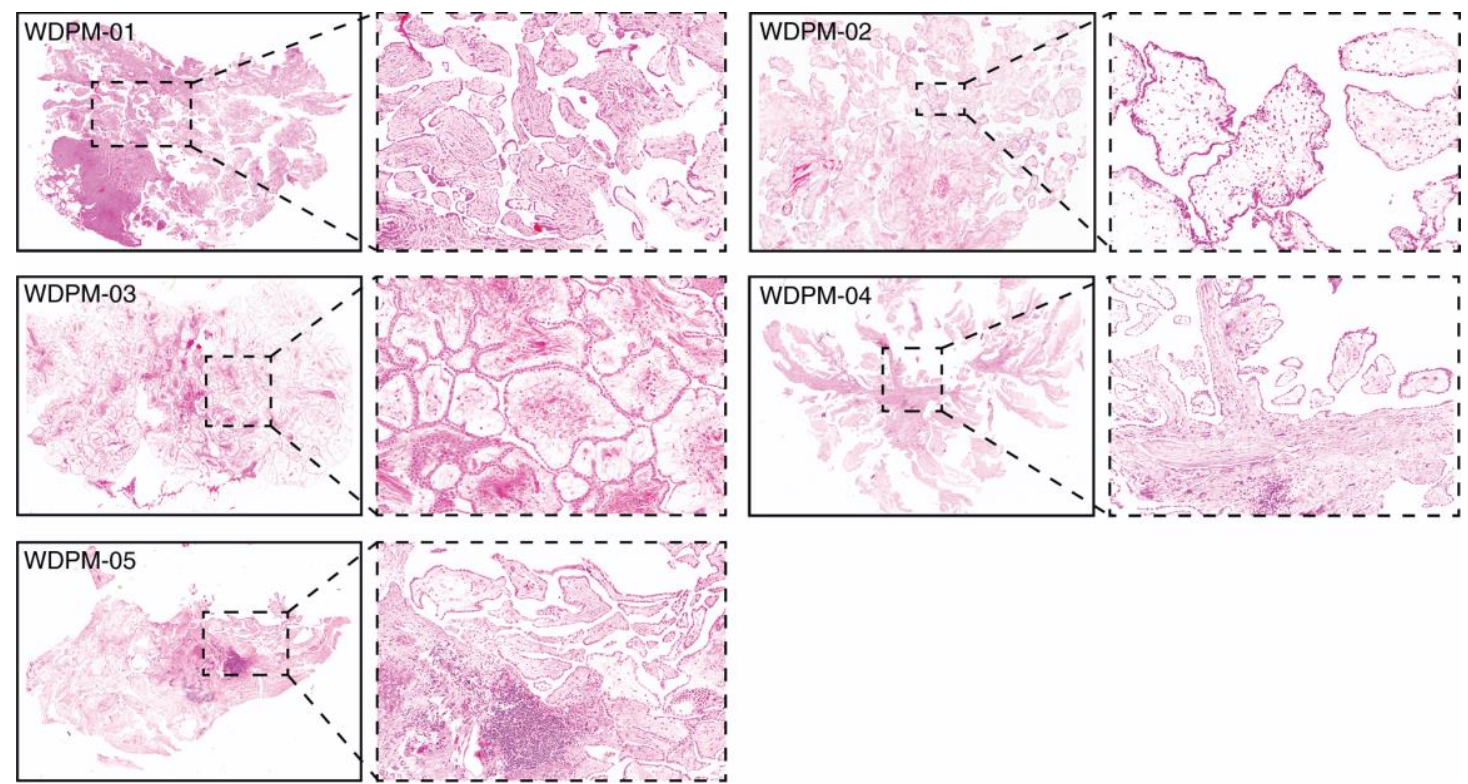

Figure 1. Histopathology of five WDPM cases used for the study. Microphotographs of histological features of WDPM stained using haematoxylin and eosin (H\&E). The panel under the dotted box represents the magnified section of the photomicrographs at $\times 20$. The lesion sites/sizes were peritoneum, site not specified, for cases WDPM-01 (3 mm), WDPM-02 (6 mm), WDPM-03 (4 mm), WDPM-04 mesentery (4 mm), and WDPM-05 omentum (4 mm).

\subsection{Mutational Landscape of WDPM}

We performed high-coverage whole exome sequencing of five WDPMs from formalin-fixed and paraffin embedded (FFPE) samples. We achieved a mean sequencing reads coverage of $87 \times-117 \times$, with at least $20-45 \%$ of targeted bases having a coverage of $100 \times$ (Table S1). Due to papillary architecture, 
the tumor cellularity of the WDPM tissues was estimated to be about 50\% (Table S2). Although the high coverage sequencing provides us an opportunity to detect higher proportions of mutations, the normal tissue admixture lowers the mutation detection sensitivity. To overcome this challenge, we implemented strict mutation filtering criteria as described in the Methods section and retained only high confident mutation calls for downstream analysis.

Analysis of the mutational patterns in WDPM revealed a strong enrichment of $\mathrm{C}>\mathrm{A}$ transversion substitution mutation (Figure 2A). Using the software deconstructSigs [13], we evaluated the characteristic mutation patterns in WDPM against the mutational signature obtained from the COSMIC mutational signature database [14]. Intriguingly, we identified consistent patterns of nucleotide substitution mutation associated with WDPM. Notably, we found that mutational signature 24 is significantly operative in all five WDPM cases (Figure 2B). In addition to this, mutational signature 21 and 28 were also observed in the WDPM cases.
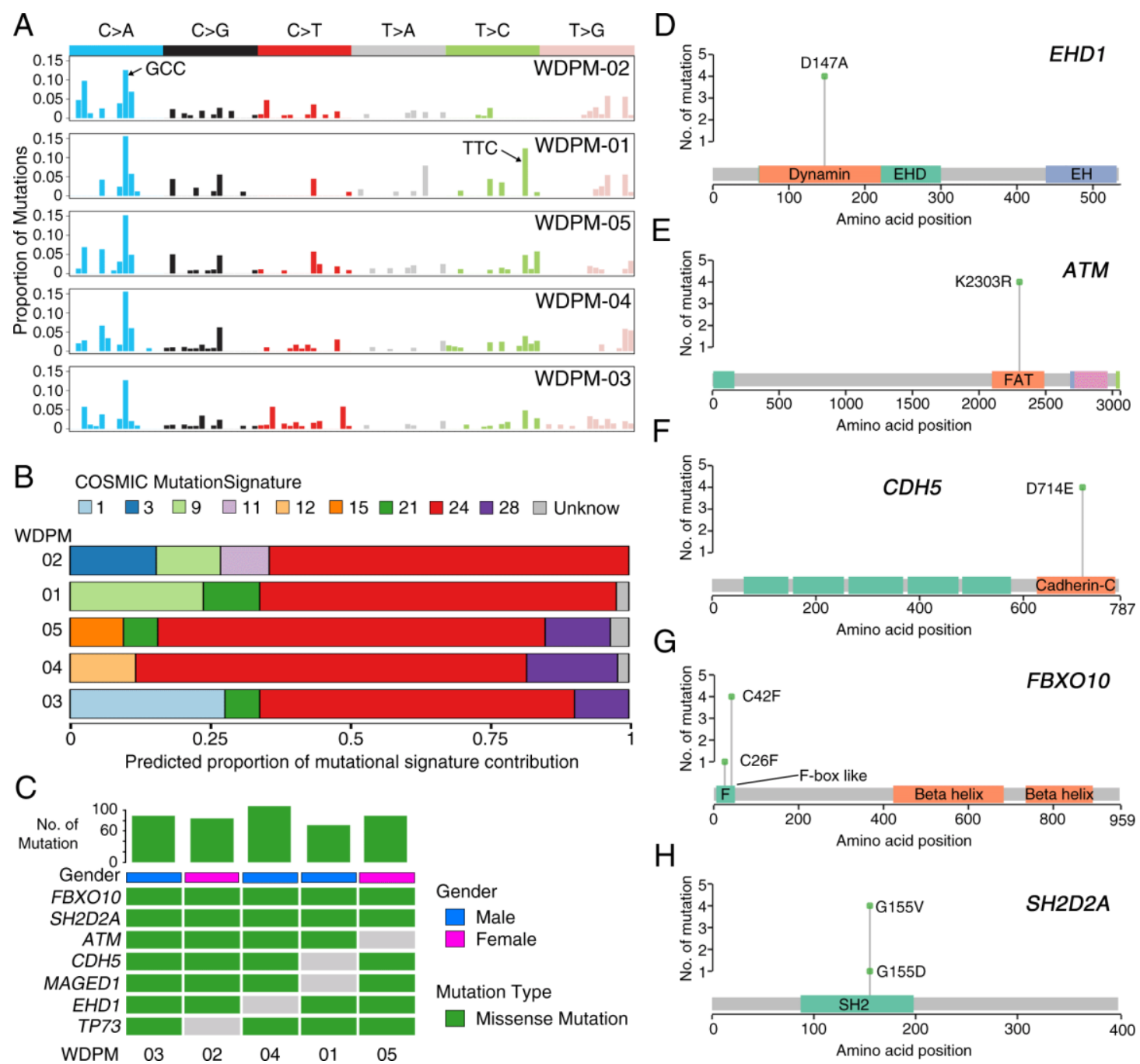

Figure 2. Landscape of mutations in WDPM. (A) Mutational signature present in WDPM. (B) Proportional contribution of different COSMIC mutational signature per sample. (C) Mutation status in WDPM. Top seven most recurrent mutations are represented in the figure. The bar plot on the top panel represents the total number of mutations detected in the respective WDPM. (D-H) Plots showing mutation distribution and the protein domains for the corresponding mutated protein.

We identified 461 unique non-silent mutations across five WDPM samples affecting 297 unique protein coding genes (Table S3). Patient WDPM-04 had the highest mutation burden and WDPM-01 had the least. Two genes-FBXO10 and SH2D2A-were mutated in all five WDPM cases, again 
displaying consistent mutational patterns (Figure 2C). Missense mutation EHD1 ${ }^{\mathrm{D} 147 \mathrm{~A}}$ in the dynamin protein domain was found in four cases (Figure 2C,D). The variant allele frequency (VAF) of EHD1 was in the range $29-43 \%$, indicating its likely clonal origin (given that the tumor cellularity of the WDPM tissues were estimated to be about 50\%) (Figure S1). Notably, we identified missense mutation in DNA-damage response gene ATM in four cases (Figure 2C,E). All four cases harbored $A T M^{\mathrm{K} 2303 \mathrm{R}}$ located in the FRAP-ATM-TRRAP (FAT) domain in the ATM protein. The VAF of ATM was also in the range $25-30 \%$, indicating its likely clonal origin (Figure S1). The gene encoding cadherin 5 (CDH5) harbored $\mathrm{CDH} 5^{\mathrm{D} 714 \mathrm{E}}$ mutations in its $\mathrm{C}$-terminus cadherin protein domain in four cases (Figure 2C,F). The VAF of $C D H 5$ was also in the range $26-38 \%$, indicating its likely clonal origin (Figure S1). We also identified missense mutation $F B X O 10^{\mathrm{C} 42 \mathrm{~F}}$ in four cases and $F B X O 10^{\mathrm{C} 26 \mathrm{~F}}$ in one case (Figure $2 \mathrm{C}, \mathrm{G}$ ). Both mutation variants of FBXO10 were present in the F-box like protein domain. The VAF of FBXO10 was also in the range $24-37 \%$, indicating its likely clonal origin (Figure S1). Similarly, we identified missense mutation $S H 2 D 2 A^{\mathrm{G} 155 \mathrm{~V}}$ in four cases and $S H 2 D 2 A^{\mathrm{G} 155 \mathrm{D}}$ in one case (Figure $2 \mathrm{C}, \mathrm{H}$ ). These variants were located in the $\mathrm{SH} 2$ protein domain. The VAF of $S H 2 D 2 A$ in WDPM-04 was $69 \%$, indicating the mutation to be clonal. The VAF of $S H 2 D 2 A$ in the rest of the four WDPMs was in the range $37-47 \%$ (Figure S1). Furthermore, we also identified mutations in MAGED1 and TP73 in each of the four WDPM cases (Figure 2C).

\subsection{Copy Number Landscape of WDPM}

The aggregate copy number aberration (CNA) profile of WDPM is shown in Figure S2. We observed 278 CNA events across all samples (Table S4). The CNA resulted in alterations of about $4-14 \%$ of the protein-coding genomes in the WDPM. Patient WDPM-02 had a high copy number burden and WDPM-03 had the least copy number burden (Figure S2). Overall, copy number profiles of the WDPM did not show many alterations (Figure S3). Notably, we found copy number gain of SETDB2 and LAST2 and copy number loss of SMARCA4 and TRAF7 in WDPM-02. We also found copy number loss of cancer genes such as CCNE1, MAF, MAFB, MYC, ZNF479, and MGMT and copy number gain of FOXA2, CDH10, and GPC5 in at least two WDPM cases.

\subsection{Signaling Pathways Dysregulated in WDPM}

To identify signaling pathways dysregulated by mutated genes in WDPM, we performed pathway enrichment analysis using the KEGG [15] pathway database (see Methods section). Our analysis revealed that WDPM mutations target different signaling pathways often dysregulated in cancer (Figure 3 and Table S5) such as pathways in cancer, focal adhesion, Vascular endothelial growth factor (VEGF) signaling, Janus kinases - signal transducer and activator of transcription (JAK-STAT) signaling, Wnt signaling, P53 signaling, apoptosis, etc. We found CDH5 mutations target cell adhesion and the leukocyte migration pathway, EHD1 mutations target endocytosis, SH2D2A mutations target the VEGF signaling pathway, ATM mutations target apoptosis and P53 signaling pathways, and TP73 targets the neurotrophin signaling and P53 signaling pathways. This indicates that the mutations identified in WDPM cases might be relevant to pathogenesis of WDPM. 


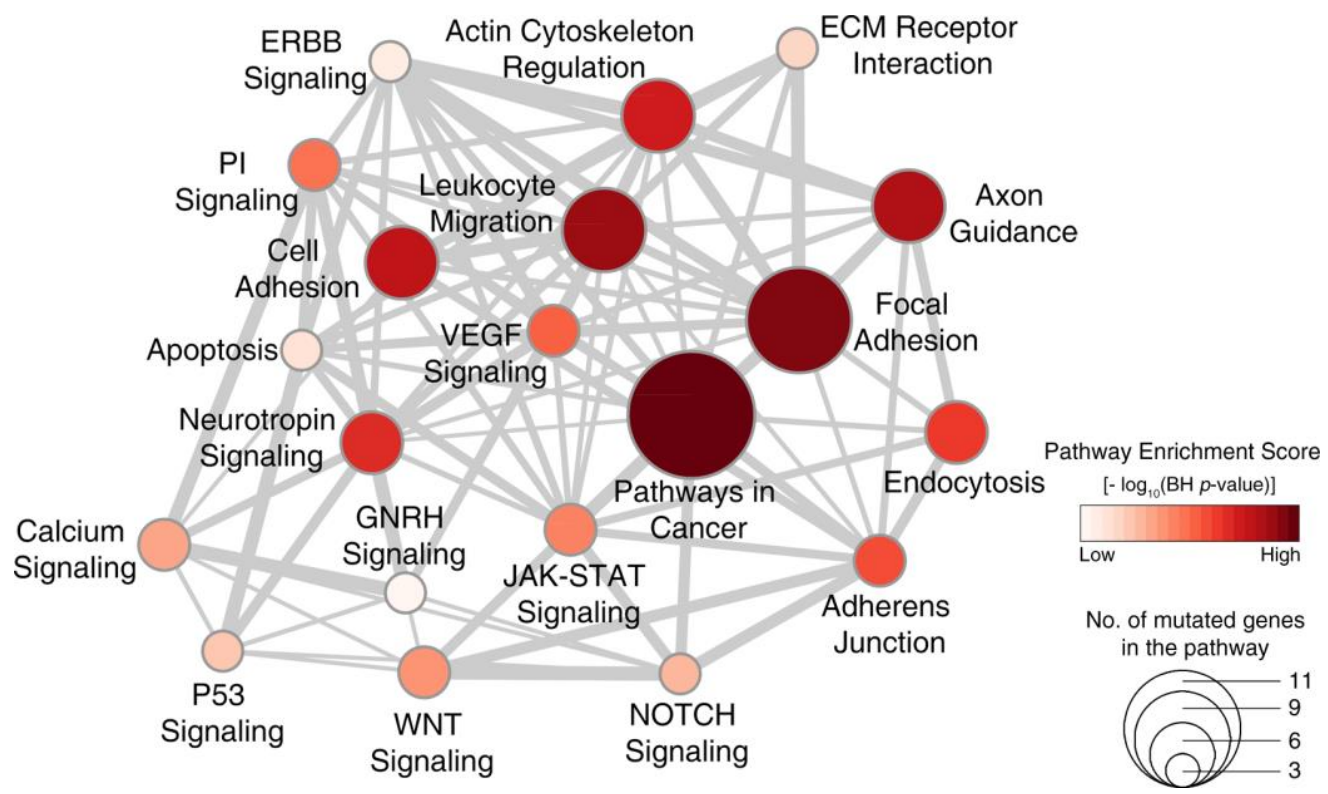

Figure 3. Signaling pathways dysregulated in WDPM. We performed pathway enrichment analysis using genes mutated in WDPM cases against the signaling pathways in the KEGG pathway database. The figure shows the top 20 pathways enriched with mutated genes in WDPM. Each circle represents a pathway, its size indicates the number of mutated genes targeting the pathway, and its color indicates the pathway enrichment score. The thickness of edges connecting two circles (pathways) is proportional to the number of mutated genes common between the two pathways. PI signaling: phosphatidylinositol signaling pathway.

\subsection{WDPM is Genetically Distinct from Malignant Mesothelioma}

Next, we compared the genomic profiles of WDPM with those of malignant peritoneal mesothelioma. For this, we leveraged the DNA sequencing data from two recently published peritoneal mesothelioma patient cohorts $[6,16]$. We first assessed the pattern of mutations in WDPM and peritoneal mesothelioma cases. Intriguingly, we observed that WDPM has a strong enrichment of C > A transversion substitution mutation (Figure 2A,B), whereas, peritoneal mesothelioma has strong enrichment of $C>T$ transition substitution mutation (Figure S4). This mutational pattern in WDPM is different from those reported in pleural $[4,5]$ or peritoneal [6] mesotheliomas.

Notably, we found WDPM specific mutations in EHD1, FBXO10, CHD5, MAGED1, ATM, and TP73 genes that were absent in peritoneal mesothelioma (Figure 4A). Although mutations in EHD1 and ATM genes were each observed in peritoneal mesothelioma, we did not find the WDPM-specific EHD1 ${ }^{\text {D147A }}$, $E H D 1^{\mathrm{A} 465 \mathrm{D}}$, and $A T M^{\mathrm{K} 2303 \mathrm{R}}$ mutations in these cases. Interestingly, in WDPM, we did not find any of the mutations in BAP1, SETD2, TP53, NF2, CDKN2A, and LAST1/2 frequently observed in malignant mesotheliomas (Figure 4A). We also did not find mutations in TRAF7 or CDC42 in WDPM, however, TRAF7 mutations were observed in several peritoneal mesothelioma cases. Furthermore, we evaluated the differences in the copy number status of genes between WDPM and peritoneal mesothelioma. We did not find any copy number loss in gene characteristics of malignant mesotheliomas such as BAP1, SETD2, PBRM1, SMARCC1, CDKN2A/B, LATS1/2, and NF2 (Figure 4B). TRAF copy number loss was observed in one WDPM case, whereas, several peritoneal mesothelioma cases harbored TRAF7 copy number alteration. 
A

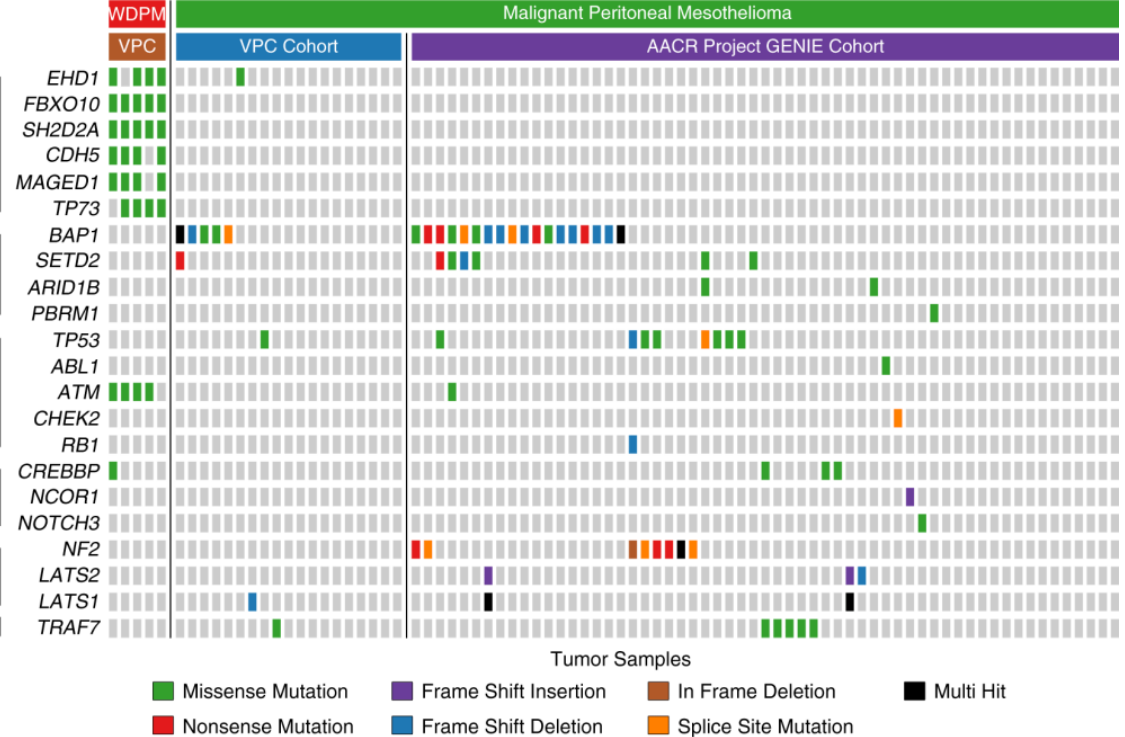

B

Nonsense Mutation

Frame Shift Deletion $\square$ Splice Site Mutation

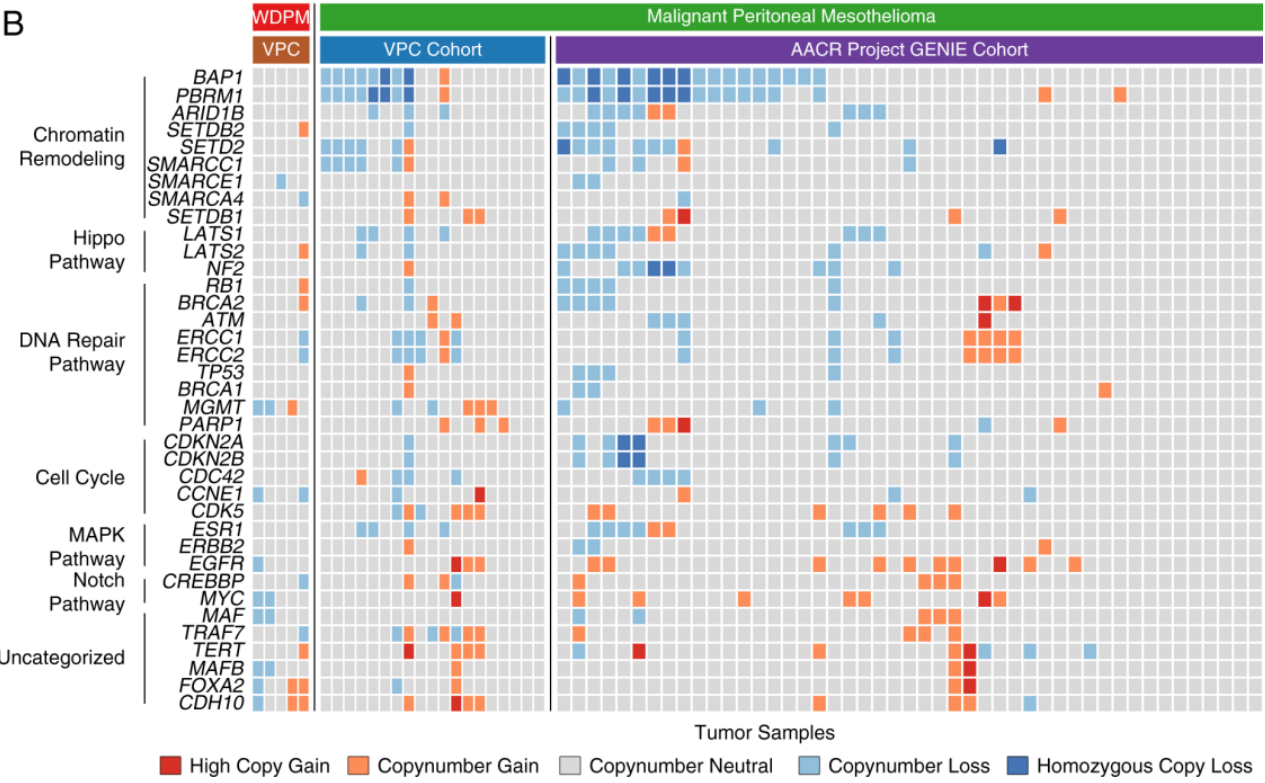

Figure 4. Genomic alterations in WDPM and peritoneal mesothelioma. We compared the genomic alteration profile of the WDPM cases to the peritoneal mesothelioma patient cohorts from two recently published studies, Vancouver Prostate Centre (VPC) cohort [6] and American Association for Cancer Research (AACR) project Genomics Evidence Neoplasia Information Exchange (GENIE) cohort [16]. (A) Oncoplot showing differences in mutation pattern between WDPM and peritoneal mesothelioma. Each column in the figure represents an individual cancer sample. (B) Oncoplot showing the copy number aberration status of WDPM and peritoneal mesothelioma. Each column in the figure represents an individual cancer sample.

\section{Discussion}

In this study, we investigated the genomic alterations found in a cohort of five WDPMs. The tumors analyzed here are clinically typical of the setting in which WDPM is most commonly found, i.e., as an incidental lesion discovered during surgery for another process, and all lesions were morphologically characteristic WDPM.

Overall, our results suggest that WDPM are distinctive lesions with their own set of genomic alterations. Given the number of mutations and the nature of the mutations found, including at least one tumor suppressor gene, TP73, and several genes that may be associated with other types 
of malignancy (ATM, CDH5, MAGED1) [17-19], WDPM clearly appears to be a functionally benign neoplasm and not a reactive process. Further, it is clear that WDPM are genetically quite different from both peritoneal and pleural mesotheliomas. Indeed, our most important finding is the lack of alterations involving BAP1, SETD2, NF2, CDKN2A, PBRM1, and SMARCC1 genes consistently mutated or deleted in malignant mesotheliomas.

We found consistent mutation patterns in five WDPMs with strong enrichment of $C>A$ transversion substitution mutation and COSMIC mutational signature 24. The WDPMs harbored distinct mutations in EHD1, FBXO10, CHD5, MAGED1, ATM, and TP73 genes either in all five or at least four out of five WDPM cases. The COSMIC mutational signature 24 has been shown to be commonly found in certain liver cancers with exposure to carcinogen such as aflatoxin [20]. However, these WDPMs were incidental findings during surgery and any prior exposure to carcinogens (either aflatoxin or asbestos) is extremely unlikely. Mutations and copy number changes in CDH5 have been previously reported in mesotheliomas [21,22] but are uncommon events and were not present in any of our reference mesothelioma datasets (Figure 3). CHD5 is known to promote intravasation and stimulates TGF- $\beta$ driven epithelial-mesenchymal transition (EMT) [23]. EHD1 regulates the endocytic recycling process. EHD1 is known to play a key role in transportation of receptors from endosomes into the endocytic recycling compartment (ERC) and from the ERC to the plasma membrane [24]. Moreover, EHD1 has been associated with cell proliferation, apoptosis, metastasis, and drug resistance in breast and non-small cell lung cancer (NSCLC) [25] but has not been reported to be abnormal in malignant mesotheliomas. FBXO10 binds to the anti-apoptotic oncoprotein BCL-2 and promotes its degradation, thereby initiating cell death in lymphomas [26]. SH2D2A is known to be involved in T-cell activation [27]. Mutations in FBXO10, SH2D2A, and TP73 has not been reported in any malignant mesotheliomas.

Our study confirms a lack of copy number alterations in BAP1, SETD2, PBRM1, SMARCC1, $C D K N 2 A / B, L A T S 1 / 2$, and NF2. Copy number loss of BAP1, SETD2, PBRM1, and SMARCC1 is often observed in peritoneal mesothelioma [6,28]. Copy number loss of $B A P 1, C D K N 2 A / B, L A S T 1 / 2$, and NF2 is frequently found in pleural mesothelioma $[4,5]$.

What is surprising in our results is the absence of the TRAF7 and CDC42 alterations reported by Yu et al. [7] and Stevers et al. [11] in WDPM and by the same group in adenomatoid tumors [29]. Alterations in TRAF7 have also been reported in malignant mesotheliomas $[4,16,30]$. However, this does not appear to be a case of tumor misclassification, since the lesion illustrated by Stevers et al. [11] is a very typical WDPM and is identical to the tumors analyzed here. The lesions analyzed by Stevers et al. [11] were also all incidental findings and 8/10 were solitary, as were ours, and the lesions for which they had follow up did not behave in a malignant fashion.

The exact reasons for the discrepancy between our study and those of Stevers et al. [11] are unclear. It is possible that the underlying populations are genetically different, particularly given the very large and diverse immigrant population in Vancouver, Canada. The analytical approach used in these two studies was also somewhat different. Stevers et al. [11] used a targeted panel consisting of 479 cancer-related genes (UCSF500 Cancer Panel) for sequencing (Illumina HiSeq 2500), whereas we used Ion AmpliSeq ${ }^{\mathrm{TM}}$ (Thermo Fisher Scientific, Waltham, MA, USA) exome sequencing which covers 18,961 genes (Ion Proton ${ }^{\mathrm{TM}}$ ). The overlap in the genes examined between these two studies is given in Figure S5A. Using a targeted panel provided Stevers et al. [11] an advantage to sequence a small number of genes at a high depth (average depth $=320 \times$, range $=33 \times-722 \times$ ), whereas we sequenced a large number of genes at a cost of sequencing depth (average depth $=102 \times$ ). Stevers et al. [11] reported 21 mutations covering 10 genes in 10 WDPM cases, whereas we identified 461 mutations covering 297 genes in 5 WDPM cases. There is no overlap of the mutated genes reported in Stevers et al. [11] and our study (Figure S5B). In fact, the UCSF500 gene panel used by Stevers et al. [11] covered only 10 mutated genes reported by our study (Figure S5C). We note that, despite high sequencing depth, no mutations in ATM (which was examined in the UCSF500 panel) were reported by Stevers et al. [11], whereas we identified consistent $A T M^{\mathrm{K} 2303 \mathrm{R}}$ mutations in 4 out of 5 WDPM cases (Figure S5C). We did 
identify a few low confidence TRAF7 mutations, but these did not pass our mutation filtering criteria (see Table S6 and Appendix A for detailed information). These differences likely indicate genomic heterogeneity in WDPM and warrants further investigation in larger patient cohort settings. Once there are sufficient cases described with consistent results, it may be possible to use a genomic approach to decide whether an equivocal case is a WDPM or a malignant mesothelioma and to base treatment on such data.

\section{Materials and Methods}

\subsection{Patient Cohort Description and Tissue Procurement}

A cohort of incidentally identified WDPM tissues $(n=5)$ were assembled from the surgical pathology archives at the Vancouver General Hospital. This study was approved by the Institutional Review Board of the University of British Columbia and the Vancouver Coastal Health (REB No. H15-00902 and V15-00902).

\subsection{Whole Exome Sequencing}

DNA from marked FFPE tissue sections (5-10 $\mu \mathrm{m}$ in thickness, 50\% WDPM cellularity) were isolated using a truXTRAC FFPE DNA Kit with Covaris Adaptive Focused Acoustics ${ }^{\circledR}\left(\operatorname{AFA}^{\circledR}\right)$ technology, which enables the removal of the paraffin from the FFPE tissue in SDS buffer while simultaneously rehydrating the tissue. The samples were treated with proteinase $\mathrm{K} 0.2 \mathrm{mg} / \mathrm{mL}$ (Roche) followed by overnight incubation at $55^{\circ} \mathrm{C}$. After post-incubation in proteinase $\mathrm{K}$, the samples were treated with RNAse and DNA extracted as per the truXTRAC FFPE DNA extraction protocol (cat\#: 520136, Covaris, Inc., Woburn, MA, USA). The amount of DNA was quantified using Qubit ${ }^{\circledR}$ dsDNA HS Assay (Thermo Fisher Scientific).

For Ion AmpliSeq ${ }^{\mathrm{TM}}$ (Thermo Fisher Scientific) exome sequencing, $100 \mathrm{ng}$ of DNA was used as input for Ion AmpliSeq ${ }^{\mathrm{TM}}$ Exome RDY library preparation, a PCR-based sequencing approach using 294,000 primer pairs (amplicon size range 225-275 bp), which covers $>97 \%$ of consensus coding sequence (CCDS) (Release 12), >19,000 coding genes, and >198,000 coding exons. Libraries were prepared, quantified using qPCR, and sequenced according to the manufacturer's instructions (Thermo Fisher Scientific). Samples were sequenced on the Ion Proton System using the Ion PI ${ }^{\mathrm{TM}} \mathrm{Hi}^{\mathrm{-}} \mathrm{Q}^{\mathrm{TM}}$ Sequencing $200 \mathrm{Kit}$ and Ion $\mathrm{PI}^{\mathrm{TM}}$ v3 chip. Two libraries were run per chip for a projected minimum coverage of 40 million reads per sample.

\subsection{Single Nucleotide Variant Calling}

We used Torrent Server (Thermo Fisher Scientific) for mapping aligned reads to the human reference genome hg19 (Torrent Mapping Alignment feature). Variants were identified using a Torrent Variant Caller plugin with the optimized parameters for AmpliSeq exome sequencing (Thermo Fisher Scientific). The variant call format (VCF) files from all samples were annotated using ANNOVAR [31].

To account for the low tumor cellularity in the WDPM samples and the absence of the matched control samples, we used strict mutation calls filtering criteria. Mutations were retained if (a) allele frequency $(\mathrm{AF})<75 \%$, (b) read quality pass $>50 \%$, (c) average heterozygosity $<0.1$, (d) mutation calls not present in dbSNP database. We filtered out all In-Dels from our variant calls. Non-silent exonic variants including non-synonymous single nucleotide variations (SNVs), stop-codon gain SNVs, stop-codon loss SNVs, splice site SNVs, and frameshift In-Dels in coding regions were retained if they were supported by more than 50 reads. Furthermore, putative variants were manually scrutinized on the Binary Alignment Map (BAM) files through Integrative Genomics Viewer (IGV) version 2.3.25 [32]. Furthermore, due to lack of matched germline control samples from the WDPM cases, we used genomic DNA samples from blood of a cohort of peritoneal mesothelioma patients as germline control samples. We filtered out any variants that were also present in these control samples [6]. In this way, we excluded any potential germline variants as well as false positive calls and obtained highly confident variants of 
WDPM. Based on the variant allele frequency (VAF), the mutations identified in WDPM were clustered into different groups using the R-package Maftools [33].

\subsection{Copy Number Aberration (CNA) Calls}

Copy number changes were assessed using Nexus Copy Number Discovery Edition Version 8.0 (BioDiscovery, Inc., El Segundo, CA, USA). Nexus NGS functionality with the FASST2 Segmentation algorithm was used to make copy number calls (a circular binary segmentation/hidden Markov model approach). The significance threshold for segmentation was $5 \times 10^{-6}$ with a minimum of 3 probes per segment and a maximum probe spacing of 1000 between adjacent probes before breaking a segment. The log ratio thresholds for single copy gain and single copy loss were set at +0.2 and -0.2 , respectively. The log ratio thresholds for homozygous gain/loss were set at +0.6 and -1.0 , respectively. The tumor BAM files were processed and compared with BAM files from a normal tissue pool as reference control. Reference reads per CN point (window size) was set to 8000 . We used the Genomic Identification of Significant Targets in Cancer (GISTIC) [34] algorithm in Nexus to identify significantly amplified or deleted regions across the genome. The amplitude of each aberration is assigned a G-score as well as a frequency of occurrence for multiple samples. The false discovery rate (FDR) q-value for the aberrant regions was set to a threshold of 0.15 .

\subsection{Mutational Signature Analysis}

We used deconstructSigs [13] software, a multiple regression approach to statistically quantify the contribution of mutational signatures for each tumor. The 30 mutational signatures were obtained from the COSMIC mutational signature database [14]. Only non-silent mutations were used to obtain the mutational signatures. In brief, deconstructSigs attempts to recreate the mutational pattern using the trinucleotide mutation context from the input sample that closely resembles each of the 30 mutational signatures from the COSMIC mutational signature database. In this process, each mutational signature is assigned a weight normalized between 0 to 1 indicating its contribution. Only those mutational signatures with a weight more than 0.06 were considered for analysis.

\subsection{Pathway Enrichment Analysis}

The mutated genes were tested for enrichment against signaling pathways present in the KEGG [15] pathway database obtained from the Molecular Signature Database (MSigDB) v6.0 [35]. A hypergeometric test-based gene set enrichment analysis was used for this purpose (https://github. $\mathrm{com} /$ raunakms/GSEAFisher). A cut-off threshold of Benjamini-Hochberg (BH) corrected $p$-value $<0.01$ was used to obtain the significantly enriched pathways. Only pathways that are enriched with at least three mutated genes were considered for further analysis.

\subsection{Peritoneal Mesothelioma Datasets}

We utilized DNA sequencing datasets of two publicly available patient cohorts of peritoneal mesothelioma-VPC cohort [6] and AACR Project GENIE Cohort [16]. We used mutation and copy number profiles from both datasets for comparison with the genomic profiles of WDPM cases. AACR GENIE project data, Version 5.0, were downloaded from https://www.synapse.org/\#!Synapse: syn7222066.

\section{Conclusions}

We have shown that WDPM are genetically distinct from malignant mesotheliomas and in our hands have a characteristic pattern of $\mathrm{C}>\mathrm{A}$ transversion substitution mutations; EHD1, FBXO10, CHD5, MAGED1, ATM, and TP73 missense mutations; as well as enrichment of COSMIC mutation signature 24. Taken in conjunction with the data from Stevers et al., these findings further reinforce the idea that WDPM should not be treated in the same fashion as malignant mesotheliomas. 
Supplementary Materials: The following are available online at http://www.mdpi.com/2072-6694/12/6/1568/s1, Figure S1. Distribution of variant allele frequency (VAF) in WDPM, Figure S2. Landscape of copy number alterations in WDPM, Figure S3. Copy number segments (log ratio) of WDPM samples, Figure S4. Mutational signature present in malignant peritoneal mesothelioma obtained from Shrestha et al., Genome Medicine 2019 [6], Figure S5. Comparison of Stevers et al. 2018 [11] with present study, Table S1. QC metric of whole exome sequencing, Table S2. Quality control statistics of WDPM samples, Table S3. Mutation profile of WDPM, Table S4. Copy number profile of WDPM, Table S5. Signaling Pathways dysregulated in WDPM, Table S6. Mutations in gene CDC42 and TRAF7 reported Stevers et al. 2018 [11] and the corresponding sequencing reads detected in WDPM cases in the present study.

Author Contributions: Conceptualization, R.S., N.N., C.C., and A.C.; methodology, R.S., N.N., and S.L.B.; software, R.S., S.V., S.A., and R.B.; validation, N.N., A.H., B.M., F.S., and S.B.; formal analysis, R.S. and N.N.; investigation, R.S., N.N., and A.C.; writing-original draft preparation, R.S., N.N., and A.C.; writing-review and editing, R.S., N.N., C.C., and A.C.; visualization, R.S.; supervision, S.L.B., Y.W., C.C., and A.C.; project administration, N.N., and S.L.B; funding acquisition, Y.W., C.C., and A.C. All authors have read and agreed to the published version of the manuscript.

Funding: This research was funded by BC Cancer Foundation, Mitacs, WorkSafe BC, Canadian Institutes of Health Research (CIHR), and Terry Fox Research Institute, and R.S. and N.N. were funded by Mitacs Accelerate Awards.

Conflicts of Interest: The authors declare no conflicts of interest. The funders had no role in the design of the study; in the collection, analyses, or interpretation of data; in the writing of the manuscript, or in the decision to publish the results.

\section{Appendix A}

Here we provide a detailed comparison between Stevers et al. [11] and the present study, which we label WDPM-VPC for convenience.

Stevers et al. [11] used a targeted panel of 479 cancer-related genes (UCSF500 Cancer Panel) for sequencing (Illumina HiSeq 2500 machine), whereas we used Ion AmpliSeq ${ }^{\mathrm{TM}}$ (Thermo Fisher Scientific, Waltham, MA, USA) exome sequencing, which covers 18,961 genes (Ion Proton ${ }^{\mathrm{TM}}$ ). The overlap in the genes examined between these two studies is given in Supplementary Figure S5A.

Using a targeted panel provided Stevers et al. [11] an advantage to sequence a small number of genes at a high depth (average depth $=320 x$, range $=33 \times-722 x$ ), whereas we sequenced a large number of genes at a cost of sequencing depth (average depth $=102 \times$ ).

Stevers et al. [11] reported 21 mutations covering 10 genes in 10 WDPM cases, whereas we identified 461 mutations covering 297 genes in 5 WDPM cases. There is no overlap of the mutated genes reported in Stevers et al. [11] and our study (Supplementary Figure S5B). In fact, the UCSF500 gene panel used by Stevers et al. [11] covered only 10 mutated genes reported by our study (Supplementary Figure S5C).

Next, we analyzed the sequencing reads covering CDC42 and TRAF7 genes in the 5 WDPM cases in this study. Stevers et al. [11] reported two unique mutations in CDC42 and six unique mutations in TRAF7. We focused on the corresponding gene regions in the WDPM cases in our study, as summarized in Supplementary Table S6.

We identified three unique very low confidence mutations in the TRAF7 gene, one in WDPM-03 and two in WDPM-01 (Supplementary Table S6). In WDPM-03, only 16 reads (out of 111 reads) supported the TRAF7 $7^{\mathrm{Y} 621 \mathrm{D}}$ mutant allele. In WDPM-01, TRAF $7^{\mathrm{N} 520 \mathrm{SD}}$ was supported by 1 read (out of 102 reads), and TRAF ${ }^{\mathrm{G} 536 \mathrm{~S}}$ was supported by 5 reads (out of 109 reads). Given that the tumor cellularity of the WDPM tissues was estimated to be about $50 \%$, the TRAF mutations mentioned above were deemed very low confidence and hence did not pass our mutation filtering criteria. The rest of the CDC42 and TRAF7 mutated regions reported by Stevers et al. [11] were identified as wild type in the WDPM cases in this study.

Thus, within the experimental settings of our study, we do not find any high confidence mutations in TRAF7 or CDC42. 


\section{References}

1. Daya, D.; McCaughey, W.T. Well-differentiated papillary mesothelioma of the peritoneum. A clinicopathologic study of 22 cases. Cancer 1990, 65, 292-296. [CrossRef]

2. Butnor, K.J.; Sporn, T.A.; Hammar, S.P.; Roggli, V.L. Well-differentiated papillary mesothelioma. Am. J. Surg. Pathol. 2001, 25, 1304-1309. [CrossRef] [PubMed]

3. Deraco, M.; Nizri, E.; Glehen, O.; Baratti, D.; Tuech, J.-J.; Bereder, J.-M.; Kepenekian, V.; Kusamura, S.; Goere, D. Well differentiated papillary peritoneal mesothelioma treated by cytoreduction and hyperthermic intraperitoneal chemotherapy-the experience of the PSOGI registry. Eur. J. Surg. Oncol. 2019, 45, 371-375. [CrossRef] [PubMed]

4. Bueno, R.; Stawiski, E.W.; Goldstein, L.D.; Durinck, S.; De Rienzo, A.; Modrusan, Z.; Gnad, F.; Nguyen, T.T.; Jaiswal, B.S.; Chirieac, L.R.; et al. Comprehensive genomic analysis of malignant pleural mesothelioma identifies recurrent mutations, gene fusions and splicing alterations. Nat. Genet. 2016, 48, 407-416. [CrossRef] [PubMed]

5. Hmeljak, J.; Sanchez-Vega, F.; Hoadley, K.A.; Shih, J.; Stewart, C.; Heiman, D.; Tarpey, P.; Danilova, L.; Drill, E.; Gibb, E.A.; et al. Integrative Molecular Characterization of Malignant Pleural Mesothelioma. Cancer Discov. 2018, 8, 1548-1565. [CrossRef] [PubMed]

6. Shrestha, R.; Nabavi, N.; Lin, Y.-Y.; Mo, F.; Anderson, S.; Volik, S.; Adomat, H.H.; Lin, D.; Xue, H.; Dong, X.; et al. BAP1 haploinsufficiency predicts a distinct immunogenic class of malignant peritoneal mesothelioma. Genome Med. 2019, 11, 8. [CrossRef]

7. Yu, W.; Chan-On, W.; Teo, M.; Ong, C.K.; Cutcutache, I.; Allen, G.E.; Wong, B.; Myint, S.S.; Lim, K.H.; Voorhoeve, P.M.; et al. First somatic mutation of E2F1 in a critical DNA binding residue discovered in well-differentiated papillary mesothelioma of the peritoneum. Genome Biol. 2011, 12, R96. [CrossRef]

8. Nemoto, H.; Tate, G.; Kishimoto, K.; Saito, M.; Shirahata, A.; Umemoto, T.; Matsubara, T.; Goto, T.; Mizukami, H.; Kigawa, G.; et al. Heterozygous loss of NF2 is an early molecular alteration in well-differentiated papillary mesothelioma of the peritoneum. Cancer Genet. 2012, 205, 594-598. [CrossRef]

9. Ribeiro, C.; Campelos, S.; Moura, C.S.; Machado, J.C.; Justino, A.; Parente, B. Well-differentiated papillary mesothelioma: Clustering in a Portuguese family with a germline BAP1 mutation. Ann. Oncol. 2013, 24, 2147-2150. [CrossRef]

10. Lee, H.E.; Molina, J.R.; Sukov, W.R.; Roden, A.C.; Yi, E.S. BAP1 loss is unusual in well-differentiated papillary mesothelioma and may predict development of malignant mesothelioma. Hum. Pathol. 2018, 79, 168-176. [CrossRef]

11. Stevers, M.; Rabban, J.T.; Garg, K.; Van Ziffle, J.; Onodera, C.; Grenert, J.P.; Yeh, I.; Bastian, B.C.; Zaloudek, C.; Solomon, D.A. Well-differentiated papillary mesothelioma of the peritoneum is genetically defined by mutually exclusive mutations in TRAF7 and CDC42. Mod. Pathol. 2019, 32, 88-99. [CrossRef] [PubMed]

12. Travis, W.D.; Brambilla, E.; Müller-Hermelink, H.K.; Harris, C.C. Pathology and Genetics of Tumours of the Lung, Pleura, Thymus and Heart; Travis, W.D., Brambilla, E., Müller-Hermelink, H.K., Harris, C.C., Eds.; WHO Press: Lyon, France, 2004; ISBN 9283224183.

13. Rosenthal, R.; McGranahan, N.; Herrero, J.; Taylor, B.S.; Swanton, C. DeconstructSigs: Delineating mutational processes in single tumors distinguishes DNA repair deficiencies and patterns of carcinoma evolution. Genome Biol. 2016, 17, 31. [CrossRef] [PubMed]

14. Alexandrov, L.B.; Jones, P.H.; Wedge, D.C.; Sale, J.E.; Campbell, P.J.; Nik-Zainal, S.; Stratton, M.R. Clock-like mutational processes in human somatic cells. Nat. Genet. 2015, 47, 1402-1407. [CrossRef] [PubMed]

15. Kanehisa, M.; Sato, Y.; Furumichi, M.; Morishima, K.; Tanabe, M. New approach for understanding genome variations in KEGG. Nucleic Acids Res. 2019, 47, D590-D595. [CrossRef]

16. AACR Project GENIE Consortium AACR Project GENIE: Powering Precision Medicine through an International Consortium. Cancer Discov. 2017, 7, 818-831. [CrossRef]

17. Rufini, A.; Agostini, M.; Grespi, F.; Tomasini, R.; Sayan, B.S.; Niklison-Chirou, M.V.; Conforti, F.; Velletri, T.; Mastino, A.; Mak, T.W.; et al. p73 in Cancer. Genes Cancer 2011, 2, 491-502. [CrossRef]

18. Wang, X.; Gao, X.; Xu, Y. MAGED1: Molecular insights and clinical implications. Ann. Med. 2011, 43, 347-355. [CrossRef] 
19. Laitman, Y.; Boker-Keinan, L.; Berkenstadt, M.; Liphsitz, I.; Weissglas-Volkov, D.; Ries-Levavi, L.; Sarouk, I.; Pras, E.; Friedman, E. The risk for developing cancer in Israeli ATM, BLM, and FANCC heterozygous mutation carriers. Cancer Genet. 2016, 209, 70-74. [CrossRef]

20. Huang, M.N.; Yu, W.; Teoh, W.W.; Ardin, M.; Jusakul, A.; Ng, A.W.T.; Boot, A.; Abedi-Ardekani, B.; Villar, S.; Myint, S.S.; et al. Genome-scale mutational signatures of aflatoxin in cells, mice, and human tumors. Genome Res. 2017, 27, 1475-1486. [CrossRef]

21. Bott, M.; Brevet, M.; Taylor, B.S.; Shimizu, S.; Ito, T.; Wang, L.; Creaney, J.; Lake, R.A.; Zakowski, M.F.; Reva, B.; et al. The nuclear deubiquitinase BAP1 is commonly inactivated by somatic mutations and 3p21.1 losses in malignant pleural mesothelioma. Nat. Genet. 2011, 43, 668-672. [CrossRef]

22. Chirac, P.; Maillet, D.; Leprêtre, F.; Isaac, S.; Glehen, O.; Figeac, M.; Villeneuve, L.; Péron, J.; Gibson, F.; Galateau-Sallé, F.; et al. Genomic copy number alterations in 33 malignant peritoneal mesothelioma analyzed by comparative genomic hybridization array. Hum. Pathol. 2016, 55, 72-82. [CrossRef] [PubMed]

23. Van Roy, F. Beyond E-cadherin: Roles of other cadherin superfamily members in cancer. Nat. Rev. Cancer 2014, 14, 121-134. [CrossRef] [PubMed]

24. Grant, B.D.; Donaldson, J.G. Pathways and mechanisms of endocytic recycling. Nat. Rev. Mol. Cell Biol. 2009, 10, 597-608. [CrossRef] [PubMed]

25. Wang, X.; Yin, H.; Zhang, H.; Hu, J.; Lu, H.; Li, C.; Cao, M.; Yan, S.; Cai, L. NF-kB-driven improvement of EHD1 contributes to erlotinib resistance in EGFR-mutant lung cancers. Cell Death Dis. 2018, 9, 418. [CrossRef] [PubMed]

26. Chiorazzi, M.; Rui, L.; Yang, Y.; Ceribelli, M.; Tishbi, N.; Maurer, C.W.; Ranuncolo, S.M.; Zhao, H.; Xu, W.; Chan, W.-C.C.; et al. Related F-box proteins control cell death in Caenorhabditis elegans and human lymphoma. Proc. Natl. Acad. Sci. USA 2013, 110, 3943-3948. [CrossRef]

27. Acuto, O.; Bartolo, V.D.; Michel, F. Tailoring T-cell receptor signals by proximal negative feedback mechanisms. Nat. Rev. Immunol. 2008, 8, 699-712. [CrossRef]

28. Yoshikawa, Y.; Emi, M.; Hashimoto-Tamaoki, T.; Ohmuraya, M.; Sato, A.; Tsujimura, T.; Hasegawa, S.; Nakano, T.; Nasu, M.; Pastorino, S.; et al. High-density array-CGH with targeted NGS unmask multiple noncontiguous minute deletions on chromosome 3p21 in mesothelioma. Proc. Natl. Acad. Sci. USA 2016, 113, 13432-13437. [CrossRef]

29. Goode, B.; Joseph, N.M.; Stevers, M.; Van Ziffle, J.; Onodera, C.; Talevich, E.; Grenert, J.P.; Yeh, I.; Bastian, B.C.; Phillips, J.J.; et al. Adenomatoid tumors of the male and female genital tract are defined by TRAF7 mutations that drive aberrant NF-kB pathway activation. Mod. Pathol. 2018, 31, 660-673. [CrossRef]

30. Sneddon, S.; Dick, I.; Lee, Y.C.G.; Musk, A.W. (Bill.; Patch, A.M.; Pearson, J.V.; Waddell, N.; Allcock, R.J.N.; Holt, R.A.; Robinson, B.W.S.; et al. Malignant cells from pleural fluids in malignant mesothelioma patients reveal novel mutations. Lung Cancer 2018, 119, 64-70. [CrossRef]

31. Wang, K.; Li, M.; Hakonarson, H. ANNOVAR: Functional annotation of genetic variants from high-throughput sequencing data. Nucleic Acids Res. 2010, 38, e164. [CrossRef]

32. Thorvaldsdóttir, H.; Robinson, J.T.; Mesirov, J.P. Integrative Genomics Viewer (IGV): High-performance genomics data visualization and exploration. Brief. Bioinform. 2013, 14, 178-192. [CrossRef] [PubMed]

33. Mayakonda, A.; Lin, D.-C.; Assenov, Y.; Plass, C.; Koeffler, H.P. Maftools: Efficient and comprehensive analysis of somatic variants in cancer. Genome Res. 2018, 28, 1747-1756. [CrossRef] [PubMed]

34. Mermel, C.H.; Schumacher, S.E.; Hill, B.; Meyerson, M.L.; Beroukhim, R.; Getz, G. GISTIC2.0 facilitates sensitive and confident localization of the targets of focal somatic copy-number alteration in human cancers. Genome Biol. 2011, 12, R41. [CrossRef] [PubMed]

35. Subramanian, A.; Tamayo, P.; Mootha, V.K.; Mukherjee, S.; Ebert, B.L.; Gillette, M.A.; Paulovich, A.; Pomeroy, S.L.; Golub, T.R.; Lander, E.S.; et al. Gene set enrichment analysis: A knowledge-based approach for interpreting genome-wide expression profiles. Proc. Natl. Acad. Sci. USA 2005, 102, 15545-15550. [CrossRef]

(C) 2020 by the authors. Licensee MDPI, Basel, Switzerland. This article is an open access article distributed under the terms and conditions of the Creative Commons Attribution (CC BY) license (http://creativecommons.org/licenses/by/4.0/). 